\title{
The influence of remanent magnetization on the thermophysical properties of modern magnetically rigid materials
}

\author{
Igor Savchenko ${ }^{*}$, Alibek Agazhanov and Dmitrii Samoshkin \\ Kutateladze Institute of Thermophysics, Siberian Branch of the RAS, 630090 Novosibirsk, Russia
}

\begin{abstract}
Temperature dependences of thermal conductivity, thermal diffusivity, enthalpy, heat capacity, density and coefficient of linear expansion of 7 brands of modern magnetically rigid materials have been studied. The experiments were carried out by dilatometry, differential scanning calorimetry, and also by a laser pulse method for determining the thermal diffusivity. First, experiments were carried out to determine the thermophysical properties of samples with remanent magnetization. After that, the samples were demagnetized by heating to temperatures exceeding the Curie temperature, after which the thermophysical properties of the demagnetized samples were measured. The obtained results allow us to state, that the presence of remanent magnetization in the materials studied does not lead to a distinguishable change in the thermophysical properties considered in this investigation.
\end{abstract}

\section{Introduction}

In the modern world strong permanent magnets are made from alloys of rare earth elements. The most commonly used alloys are Nd-Fe-B and Sm-Co systems with various alloying additives. These materials have record values of remanent magnetization and coercive force. Their magnetic properties are actively investigated, but other properties are practically not studied. An investigation of the thermophysical properties of magnetically rigid materials is an interesting problem for many reasons, one of which is the possibility of discovering new magnetothermal phenomena. The present work is devoted to clarify the question of the influence of residual magnetization on density, coefficient of thermal expansion, thermal conductivity, thermal diffusivity, specific heat and enthalpy of some industrial brands of magnetically rigid materials used for the manufacture of permanent magnets.

\section{Investigated materials}

The experiments were carried out with samples of three brands of neodymium magnets: $\mathrm{N} 35 \mathrm{M}, \mathrm{N} 35 \mathrm{H}$ and $\mathrm{N} 35 \mathrm{SH}$. This is hard magnetic materials containing as a main component

\footnotetext{
* Corresponding author: savchenko@itp.nsc.ru
} 
the crystalline phase of $\mathrm{Nd}_{2} \mathrm{Fe}_{14} \mathrm{~B}$ type. The technology of their manufacture is described in [1], and their technical characteristics are listed in [2]. According with the information provided by the manufacturer the samples have the following chemical structure, namely $\mathrm{Fe}$ $\sim 71 \%$, Nd $\sim 24-27 \%$, Dy $\sim 0.5-2.5 \%$, Co $\sim 1 \%$, B 1\%. According to the composition various brands differ the content of dysprosium, which is $0.5,1$ and $2.5 \%$ for brands $\mathrm{N} 35 \mathrm{M}$, $\mathrm{N} 35 \mathrm{H}$ and $\mathrm{N} 35 \mathrm{SH}$, respectively. From the viewpoint of known properties, the specimens of different brands distinguish range of the operating temperatures and the value of the coercive force. For our compounds we have that the more of dysprosium in the sample, the higher the coercive force and the upper limit of the permitted temperature interval.

Samples similar in composition to the intermetallic compounds $\mathrm{SmCo}_{5}$ and $\mathrm{Sm}_{2} \mathrm{Co}_{17}$ were also investigated, namely two brands of each type: YX18, YX24 and YXG22, YXG30, respectively. According to the manufacturer's data [3], the samples of different brands differed in the value of the maximum magnetic energy, from the point of view of chemistry the differences are due to small additions of $\mathrm{Pr}, \mathrm{Fe}, \mathrm{Cu}$ and $\mathrm{Zr}$. Compared with magnets based on compounds of the Nd-Fe-B system, samarium compounds with cobalt have higher values of maximum operating temperature $(\sim 500-700 \mathrm{~K})$ and Curie temperature $(\sim 950-1100 \mathrm{~K})$.

\section{Experimental technique}

The density of the samples at room temperature was determined by hydrostatic weighing using an AND-GH 300 scales [4]. The thermal expansion was investigated on horizontal dilatometer DIL-402C [5] produced by the German company NETZSCH with holder and pushrod made of fused silica. Technique of carrying experiments was described in detail in [6]. The specific heat coefficient $\left(C_{P}\right)$ was investigated by the method of differential scanning calorimetry (DSC) using the automated experimental setup NETZSCH DSC 404 F1 [7]. Measurements were carried out in low (190-375 K) and high temperature $(318-800 \ldots 1270 \mathrm{~K})$ ranges with a heating rate of $2 \mathrm{~K} / \mathrm{min}$ and $5 \mathrm{~K} / \mathrm{min}$, respectively, in flowing argon atmosphere $(20 \mathrm{ml} / \mathrm{min})$. The used calibration samples were sapphire and $12 \mathrm{Kh} 18 \mathrm{~N} 10 \mathrm{~T}$ stainless steel for the low temperature and the high temperature ranges, respectively. All the investigated samples of magnets were placed in platinum crucibles. The thermal diffusivity was measured by NETZSCH LFA-427 [8]. The experiments were carried out in an argon atmosphere. The alumina sample holder and the graphite cap were used. Calculation of the thermal diffusivity coefficient was implemented taking into account the heat losses from all surfaces of the sample according to the model [9]. Corrections to the finite duration of the laser pulse and its real shape were introduced [10]. The error in the experimental determination of density, heat capacity and thermal diffusivity in the whole research temperature range does not exceed $0.5 \%, 4 \%$ and $5 \%$, respectively. Data on enthalpy were calculated from the results of experiments with DSC, and the thermal conductivity was calculated using the results of measurements of thermal diffusivity, density and heat capacity.

\section{Results and discussion}

In Fig. 1, 2, 3 comparisons of the measurement results of extension, heat capacity and thermal diffusivity for magnetized and demagnetized samples of the N35M brand are presented. The results are similar for magnets of other brands. It can be seen that the results obtained on samples with different degrees of magnetization are in good agreement with each other. This suggests that the dependence of the studied thermophysical properties on the residual magnetization is too weak to be reliably fixed by our instruments. 


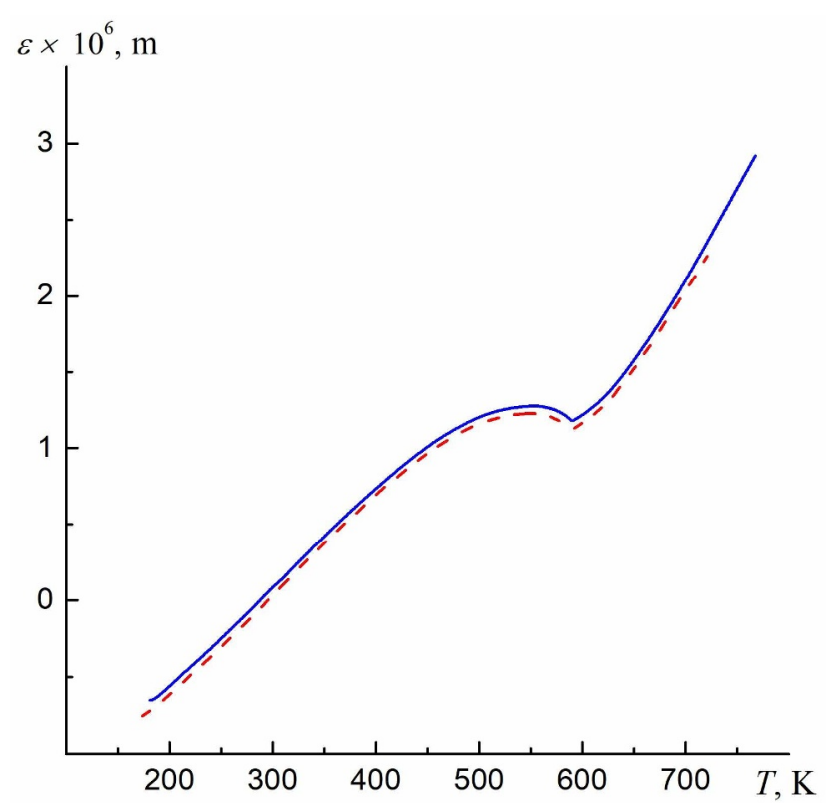

Fig. 1. Comparison of the measurement results of the extension of the N35M magnetically rigid material. The red broken line marks the results obtained on samples with residual magnetization at room temperature, the blue solid line marks the results for demagnetized samples.

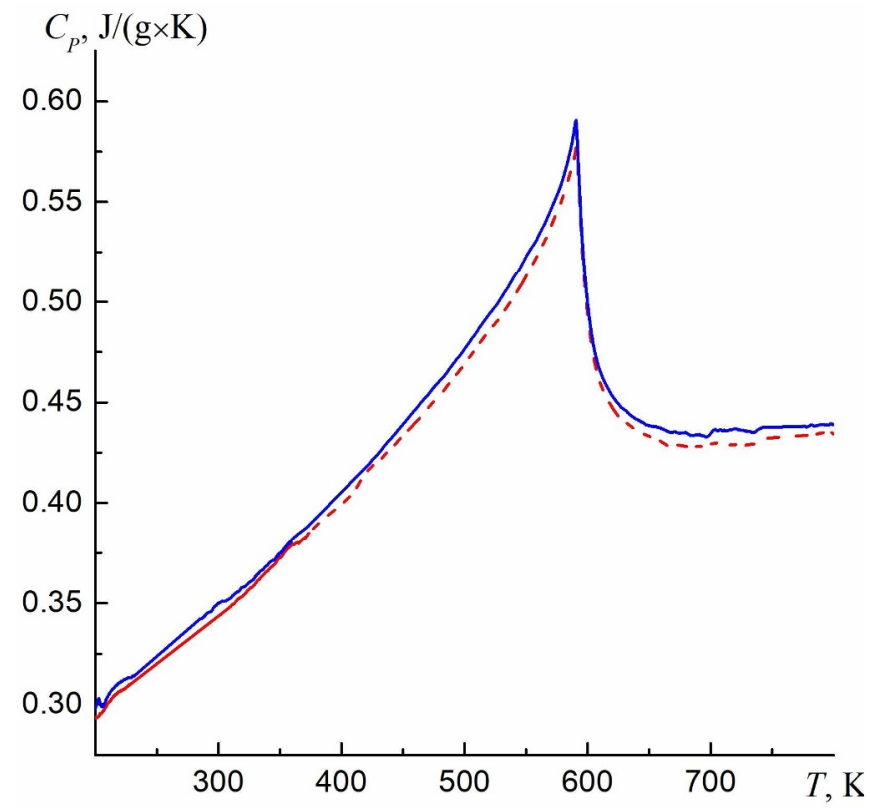

Fig. 2. Comparison of the measurement results of the heat capacity of the N35M magnetically rigid material. The red broken line marks the results obtained on samples with residual magnetization at room temperature, the blue solid line marks the results for demagnetized samples. 


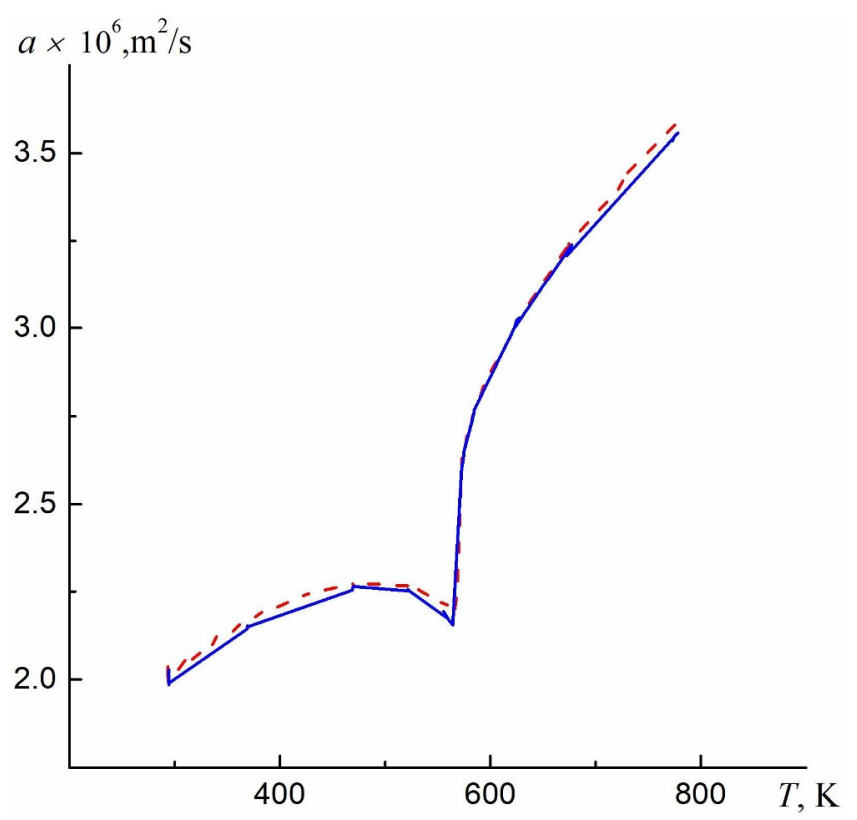

Fig. 3. Comparison of the measurement results of the thermal diffusivity of the N35M magnetically rigid material. The red broken line marks the results obtained on samples with residual magnetization at room temperature, the blue solid line marks the results for demagnetized samples.

\section{References}

1. Patent No: US 8182618 B2

2. magnit54.com/biblioteka/harakteristiki_neodimovyh_magnitov

3. http://magnet-prof.ru/index.php/harakteristiki-magnitnogo-materiala-smco.html

4. http://a-and-d.ru/product/gh-300

5. https://www.netzsch-thermal-analysis.com/ru/produkty-reshenija/dilatometrija/dil-402expedis-classic/

6. Yu.M. Kozlovskiy, S.V. Stankus, High Temperature, 52, 536 (2014)

7. https://www.netzsch-thermal-analysis.com/ru/produkty-reshenija/differencialnajaskanirujushchaja-kalorimetrija/dsc-404-f1-pegasus/

8. https://www.netzsch-thermal-analysis.com/ru/produktyreshenija/temperaturoprovodnost-i-teploprovodnost/lfa-427/

9. J.A. Cape, G.W. Lehman, Journal of Applied Physics, 34, 1909 (1963)

10. J. Blumm, J. Opfermann, High Temperatures-High Pressures, 34, 515 (2002) 